\title{
Aerobic exercise improves quality of life and CD4Cell counts in HIV seropositives in Nigeria
}

\begin{abstract}
Background: Studies that examine the effectiveness of exercise on quality of life and CD4 cells of HIV population are not easily available in developing countries.

Purpose: This study was therefore aimed at investigating the effect of 12 weeks aerobic exercise on quality of life and CD4 cells of HIV seropositives in Nigeria.

Methods: Using convenient sampling, 91 volunteer and willing HIV infected persons were enrolled in the study. Based on inclusion criteria, 82 were eligible for the study, and randomly assigned to control and experimental groups. After determining the baseline values of the variables, the experimental group participated in moderate intensity aerobic exercise for 12 weeks. Weekly lectures were organized for the control group. Attrition rate of $22 \%$ was recorded at the end the study, leaving 32 participants in each group. The variables were re-assessed at the end of the Descriptive statistic summarized the sociodemographic characteristics of the participants. Paired Student t-test and Student t-test for two independent samples analyzed the significant difference in mean values of the variables.

Results: Mean ages in years of the control and experimental groups were39.38 \pm 10.03 and $40.84 \pm 10.05$ respectively, and that of the 64 participants was $39.57 \pm 10.13$. There was significant improvement $(\mathrm{p}<0.05)$ in the variables between pre- and post-tests in the experimental group. There was insignificant change $(p>0.05)$ in the four domains of quality of life of the participants, and significant reduction $(p<0.05)$ in the other four domains of this variable between pre- and post-tests in the control group, in CD4 cell counts, significant improvement was recorded. The variables at the end of the study showed significant difference $(\mathrm{p}<0.05)$ between both groups
\end{abstract}

Conclusion: Quality of life and CD4 cells of HIV seropositives in the experimental group improved significantly after 12 weeks moderate intensity aerobic exercise.

Keywords: Aerobic exercise, Quality of life, CD4 cell counts, HIV seropositives, Convenient sampling, Attrition rate
Volume 5 Issue 3 - 2017

\author{
Maduagwu Stanley M,' Gashau Wadzani, ${ }^{2}$ \\ Balami Adamu, ${ }^{3}$ Kaidal Amina, ${ }^{3}$ Oyeyemi AY, ${ }^{4}$ \\ Denue Ballah Akawu, ${ }^{2}$ Umeonwuka Chuka \\ Ifeanyi, ${ }^{4}$ Akanbi OA' \\ IDepartment of Physiotherapy, University of Maiduguri Teaching \\ Hospital, Nigeria \\ 2Department of Medicine, University of Maiduguri, Nigeria \\ ${ }^{3}$ Department of Physical and Health Education, University of \\ Maiduguri, Nigeria \\ ${ }^{4}$ Department of Medical Rehabilitation, University of Maiduguri, \\ Nigeria
}

Correspondence: Stanley M Maduagwu, Department of Physiotherapy, University of Maiduguri Teaching Hospital, Maiduguri, Nigeria, Tel +234803499820 7, +234708663899 8, Email stmanofpeace@gmail.com

Received: December 19, 2016 | Published: March 20, 2017

\section{Introduction}

The first case of acquired immunodeficiency syndrome (AIDS), the progeny of human immunodeficiency virus (HIV) was diagnosed in Lagos, Nigeria in 1985 in a teenage girl aged 13 years and reported officially in 1986. ${ }^{1,2}$ Prior to this period, Nigerians believed that AIDS was a foreign and white man's disease, incapable of affecting the natives. ${ }^{3}$ Nigerians were therefore skeptical and under estimated the presence of AIDS in the country, consequently nothing was done at that earlier stage to curb the spread of the ailment. ${ }^{4,2}$ In this state of doubt, Nigeria, the most populous black nation, projected at $>160$ million by the World Bank in $2011^{5}$ found herself dealing with a disease which gradually transformed to an epidemic and currently placed her as the second highest burden of HIV/AIDS worldwide. ${ }^{6}$ By 2011, an estimated 3.2 million Nigerians were living with HIV with about 2.4 million children orphaned by AIDS. ${ }^{6}$ HIV impacts greatly on every facet of lives of non infected and infected individuals. It does not affect only the infected person, but also his/her family, community and the entire society. ${ }^{7}$ In families, companionship and income are lost with imminent poverty, and increase in the number of orphans and widows. At community and national levels, there is loss of productivity because of absenteeism from work due to ill health caused by the virus. ${ }^{8}$ As a result of this chain reaction triggered by HIV infection, coupled with diminishing immune function (the prime effect of the virus), the victims undoubtedly face several psychological problems with distorted mental health, and subsequently low quality of life. Orlando et al. ${ }^{9}$ observed that HIV infected persons have higher episodes of psychological disorders which negatively alters their quality of life continuum compared to uninfected individuals. RiveroMendez et al. ${ }^{10}$ reported that depression, anxiety, fatigue, fear/worries, difficult concentration, memory loss and insomnia are some ofthe major psychological symptoms resulting to low quality of life among HIV infected individuals. Anxiety and depression were identified as the most culpable symptoms that lead to low quality of life among HIV infected population. ${ }^{11}$ Moderate to severe levels of depression were reported by Heckman and colleagues ${ }^{12}$ in $25 \%$ of HIV seropositives whose ages ranged between 50 years and above. Also Penzak et al. ${ }^{13}$ found $22 \%-45 \%$ prevalence rate of depression among HIV infected persons. Invariably these symptoms coupled with resultant reduction in quality of life lowers the overall state of mental wellbeing; sense of belonging and life satisfaction of HIV infected persons.

In addition to reduced quality of life, HIV primarily selects and attacks the CD4 cells which are the key coordinators of other cells of the immune system in boosting the immune functions. ${ }^{14}$ According to Jaggers, ${ }^{15} \mathrm{HIV}$ selectively attacks CD4cells because of specific surface receptors inherent in them. These receptors are known to facilitate cellular fusion with HIV glycoprotein complex called glycoprotein 120 (gp120). ${ }^{15}$ Apart from the direct impact of HIV on CD4cells, stress has been reported to reduce CD4cell counts. ${ }^{16}$ Stress is one of the aftermaths of anxiety and depression, which are indicators of low quality of life and the most common symptoms experienced by HIV population. ${ }^{11}$ Thus, the state of wellbeing of HIV infected persons may affect the levels of CD4cell counts in the immune system. For 
instance, Nokes and Kendrew ${ }^{17}$ established a correlation between HIV infection and insomnia. Insomnia is defined as insufficient quantity or quality of sleep which according to Darko et al. ${ }^{18}$ may increase the stress level of HIV infected persons; this in turn precipitates more real-life stressors such as unemployment and decreased quality of life. Having enough sleep is known to improve and boost immune functions. Advent of highly active antiretroviral therapy (HAART), about three decades ago brought succor to HIV infected persons. The therapy has the ability to inhibit the growth and replication of $\mathrm{HIV}$, and partially reconstitute the adaptive immune system. ${ }^{19}$ As a result, the hitherto dreaded disease was transformed to a manageable chronic illness. Notwithstanding this transformation, HAART's arrival was accompanied with considerable adverse effects. One of the consequences of these undesirable effects is the reduction in quality of life components of HIV infected individuals with inherent reduction in CD4cell counts, just as the HIV infection itself. ${ }^{20}$ Post HAART era also saw the emergency of a condition known as lipodystrophy which is associated with physiological changes that disrupt the body's functions in producing, utilizing and distributing fat with resultant alteration in body composition. ${ }^{19,20}$ Hence, this alteration in body composition results to haphazard distribution of fat in the body. These changes in body composition impact negatively on the quality of life of HIV infected persons. ${ }^{20}$ Similarly, the complex and demanding schedules for HAART may interfere with the performance of daily life tasks, including personal care and employment. This interference, as reported by O'Brien et al. ${ }^{21}$ may reveal the individual's HIV status, which may result in some form of stigmatization and discrimination that may subsequently lead to an array of social deprivation with inherent low quality of life. Tiozzo et al. ${ }^{19}$ reported that this gamut of psychological problems resulting to low quality of life with concomitant stress and reduced CD4 cells in this population may be improved by engaging in regular exercise.

Several studies ${ }^{22-26}$ had examined the effect of exercise on quality of life in HIV infected population. Four of these studies ${ }^{22-26}$ reported significant positive changes in quality of life of HIV infected persons post exercise therapy, while Birk et al. ${ }^{25}$ found favorable changes which were insignificant. Also, exercise has been reported to enhance CD4 cell counts by reducing negative emotional states and modulating levels of endogenous opiates and stress hormones. ${ }^{27}$ In addition, Velkovic ${ }^{28}$ reported that moderate intensity aerobic exercise has the ability to stimulate the formation of certain antibodies that prevent some potent HIV protein molecules (glycoprotein \{gp $\} 120)$ from attaching to receptor sites of CD4 cells. By this process, Djordjevic et al. ${ }^{29}$ posited that the damaged immune system is reconstituted and HIV disease progression to AIDS is slowed down..$^{29}$ Thus, no report so far has shown any negative outcome of exercise on quality of life and CD4cell counts of HIV infected persons. However, none of these studies on impact of exercise on quality of life and CD4cells of HIV infected persons seems to have been reported from Nigeria. This paucity of either empirical or anecdotal data on effect of exercise on quality of life and CD4 cells of HIV infected persons prompted the authors to conceive and design this study. The objective of this study was therefore to examine the effect of 12 weeks moderate intensity treadmill aerobic exercise on quality of life and CD4cells of HIV seropositives in Nigeria.

\section{Materials and methods}

\section{Participants}

The study population was HIV seropositives on HAART whose CD4 cell counts were not below 200cells $/ \mathrm{mm}^{3}$. These individuals at the period of study were attending antiretroviral (ARV) clinic at a teaching hospital in Nigeria. In 1986, the federal government of Nigeria designated this hospital as a center of excellence in immunology and infectious diseases. Presently, it has a capacity of 530 beds and serves about 25 million people across Nigeria, and the neighboring countries of Cameroun, Chad and Niger republic. On the average, the monthly attendance of persons infected with HIV and AIDS to the ARV clinic at the hospital is about 6000 . The sample for the study was based on the availability of volunteer and eligible participants. This is due to the peculiarity of the study population which attracts special attention and therefore needs confidentiality owing to the nature of the infection, and the way HIV and AIDS are perceived in our environment. For this reason, convenient sampling technique was employed to enroll volunteers. In all, 91 HIV infected persons willingly enrolled to participate after being sensitized on the importance of the study.However, based on exclusion and inclusion criteria, 82 participants were found eligible for the study. Five willing participants had CD4 cell counts less than 200cells/mm3, two were pregnant women, one was a nursing mother and another had peripheral neuropathy with weakness of the lower limbs. These nine persons were therefore excluded from the study.

\section{Study design}

This study was construed based on pretest - post-test control group design. Hence, the 82 eligible participants were randomly selected and either assigned to an experimental or a control group. At the end of this random assignment, each group had 41 participants. By the end of the 8th week of the aerobic exercise training program, the study recorded an attrition rate of $22 \%$, leaving each group with 32 participants until the end of the study. In other words, 64 participants completed the aerobic exercise training program. This relative high attrition rate could be partly attributed to the insurgency (during the period of study) in the part of Nigeria where the study was conducted. Basedon this, some participants might had relocated to other parts of the country.

\section{Procedure for data collection}

The Research and Ethical Committee of the teaching hospital gave approval for the study. One of the authors (MSM) sat for and passed Biomedical Research Basic/Refresher (Reference ID: 11844307) course in order to satisfy Collaborative Institutional Training Initiative (CITI) requirements for researchers involved primarily in Biomedical research with humans. The authors sensitized the population attending ARV clinic on the need to participate in the study and explained to them the objectives, protocols and benefits of the study. After the explanation, the aforementioned 91 participants willingly enrolled for the study, nine were however excluded from the study. Two versions of informed consent forms (English Language version and translated Hausa Language version) were provided for the remaining 82 participants. Those who could read, understand and write English Language signed the informed consent forms.

Participants who were not literate in English Language gave their consents by thumb printing on the Hausa Language translated version. Hausa Language is the major language in the locality where the study was conducted and interestingly all participants understand this language. The participants were screened for eligibility by two research physicians at the ARV clinic, who also conducted physical examination on each participant. Participants either completed or were assisted to complete the bio-data form. To assess quality of life of the participants, we adopted ResearchAndDevelopment (RAND) 36-item short form (SF-36) Health Survey Questionnaire. This tool comprises eight domains on physical and mental components, and was used by past similar studies. ${ }^{19,30}$ Each of the eight domains contains 
items ranging from two to 10 . For instance, the physical functioning domain has 10 items and elicits questions on how much the present health of the participants limits their activities (listed on each of the items) on a typical day. Role limitation due to emotional problems domain consists of three items. This domain asks the participants whether they have had any of the problems (listed on each of the three items) with their work or other regular daily activities as a result of any emotional problems (such as feeling depressed or anxious) during the past 4 weeks. The social functioning and bodily pain domains encompass two items each and the items ask questions relating to their respective domains. The reliability of the eight domains of SF36 has been estimated using test-retest method. With rare exceptions, published reliability statistics of each domain have exceeded the minimum standard of 0.70 recommended for measures used in group comparisons in more than 25 studies ${ }^{31}$ most have exceeded $0.80 .{ }^{32}$ The content validity of the SF-36 has been compared to that of other widely used generic health surveys. ${ }^{32}$ SF-36 scales have been shown to achieve about $80-90 \%$ of their experimental validity in studies involving physical and mental health conditions $\left({ }^{33} \mathrm{~A}\right.$ study by Burgess et al. ${ }^{34}$ had established the reliability and validity of this instrument in populations with HIV.

RAND approves without written permission from users, the adoption and modification of some wordings of the items of SF-36 Health Survey Questionnaire which may seem difficult to understand by the participants before administration. Based on the terms and conditions stipulated by RAND for researchers who adopt the questionnaire, we modified some wordings in nine items to suit our environment, as well as for easy comprehension by the participants. The modified questionnaire was translated to Hausa Language version by an expert and a lecturer in Hausa Language for participants who could neither read nor understand English Language.

This translated version served two purposes:

I. Participants who could read and understand Hausa Language completed the questionnaire themselves.

II. For participants who could not read but understood Hausa Language, one of the authors who writes, speaks and understands Hausa Language read this version and assisted them to complete the instrument. The instrument was administered to the participants at baseline and after 12 weeks of the exercise training program.

A laboratory scientist who specializes in Immunology collected blood samples from the participants and transferred these samples to tripotassium ethylene diaminetetracetic acid (K3EDTA) bottles for onward analysis of CD4 cell counts by flow cytometry (Partec GmbH 2005 model, Munster, Germany) using monoclonal antibody panels technique.This method is based on adding monoclonal antibodies to a blood sample and running the fluid through a light source, usually a laser beam. This analysis was performed at baseline, ends of 4th and 8th weeks, and after the 12th week of the aerobic exercise training by the same laboratory scientist in order to minimize error and ensure reliability. On each occasion, the scientist collected the blood samples in the morning following an overnight fast of 10-12 hours.

\section{Exercise protocol}

The intervention group for this study was subjected to 12 weeks moderate intensity treadmill aerobic exercise, three times per week for 40 minutes per session at the physical therapy department of the hospital where the study was conducted. The 40 minutes exercise session comprised 10 minutes of warm up phase which included stretching exercises among others, 20 minutes conditioning/training phase performed on computerized treadmills which are equipped with the necessary parameters, such as speed per hour, distance covered per time, inclination etc, and finally, 10 minutes cool down phase. The inclination of the treadmills was at 00 to reduce the effect of the stress of exercise on the participants because of their HIV status which predisposes them to early fatigue. Digital heart rate monitor (Port Washington, New York, USA) was used to observe the target training heart rate of each participant. The starting exercise intensity/target training heart rate (TTHR) at 1 st week-2nd week was $50 \%$ of heart rate reserve (HRR), this was gradually increased to $75 \%$ of HRR at the last phase (11th - 12th week) of the exercise protocol. These chosen parameters (mode, frequency, duration and intensity) for this study were in line with those used in previous studies. ${ }^{19,22,24,30}$ The Karvonen method was used to determine the TTHR for the participants. Calitz ${ }^{30}$ reported that most HIV studies use this method to determine the TTHR of HIV population. The training exercise protocol was supervised by two physical therapists, an exercise physiologists and a physician who are among the authors of this article.

The control group participants were instructed not to engage in any form of exercise apart from their routine daily activities throughout the period of study. The lead author telephoned them every 3 weeks to maintain contact and promote their interest in the study. In addition, the authors organized documented lectures for this group once a week on issues pertaining to nutrition, meaning of HIV and AIDS, relationship and differences between HIV and AIDS, mode of HIV transmission and its prevention, and adherence to ARV therapy.

\section{Statistical analyses}

Frequency counts and percentages were employed as descriptive statistics to summarize socio-demographics of the participants. A bar chart was used to present the CD4cell counts at baseline, 4th and 8th weeks, and at the end of the 12th week of the study. The significant difference between the variables at baseline and 12th week in the intervention group, as well as in the control group was compared using paired Student t-test. Student t-test for independent samples analyzed significant difference in the variables between the control and intervention groups at baseline and at the end of the study. All analyses were executed using Statistical Package for the Social Sciences (SPSS) version 20.0 software (SPSS Inc., Chicago, Illinois, USA). Alpha value was set at $\mathrm{p}<0.05$ level of significance.

\section{Results}

Table 1 displays the socio-demographic characteristics of the 64 HIV infected persons who participated from the beginning of the study to the end. The mean ages in years of the participants in the control and experimental groups were $39.38 \pm 10.03$ and $40.84 \pm 10.05$ respectively. Their ages ranged from 21-60 and 20-60 for the control and experimental groups respectively. The mean age in years of the 64 participants was $39.57 \pm 10.13$, with age range of between 21 and 60 years. In both groups, the female participants $(59.4 \%$ control versus $68.8 \%$ experimental) were more than their male counterparts. Majority of the participants were married in either of the groups, (62.5\% in the control and $68.7 \%$ in the experimental). Most (59.4\%) participants in the control group had secondary education or above, while in the experimental group those without formal education predominated $(43.8 \%)$. In either group, participants that had jobs were in preponderance $(81.2 \%$ control; $68.7 \%$ experimental). The only Figure1 in this study reveals the mean CD4 cell counts of the participants in the control and experimental groups. This figure portrays that the mean CD4 cell counts of the participants in the experimental group increased exponentially from the baseline to the 
end of the study compared to that in the control group. There was no significant difference $(p>0.05)$ in the baseline values of the variables (i.e. the eight domains of quality of life and CD4 cells) between the control and experimental groups. By implication, the values of these variables were similar in the two groups at the commencement of the study. Thus, the observed changes in these variables at the end of this study could be attributed to the moderate intensity treadmill aerobic exercise intervention. Table 2 summarizes this observation. The quality of life domains and CD4 cell counts of the participants in the experimental group after the end of the study were significantly higher $(p<0.05 ; p$-value $=0.000)$ than the values before the beginning of the study. In the control group, the result reveals that pre-and post-tests mean scores of physical functioning, role limitation due to physical health, role limitation due to emotional health and bodily pain of the participants did not differ significantly $(p>0.05)$. In contrast, the mean scores of energy/fatigue, emotional well being, social functioning and general health were significantly lower $(p<0.05)$ at the end of the study. The CD4cell counts of the participants in the control group at the end of the study improved significantly $(p<0.05)$, although not as observed in those of the experimental group (Table 3). Table 4 depicts post - test comparison of quality of life variables and CD4 cell counts of the participants in the control and experimental groups.

Table I Socio-demographic Characteristics of the Participants $(n=64)$

\begin{tabular}{|c|c|c|}
\hline Variables & $\begin{array}{l}\text { Control Group } \\
n=32\end{array}$ & Experimental Group $n=32$ \\
\hline Age (years) & $39.38 \pm 10.03(21-60)^{*}$ & $40.84 \pm 10.05(20-60)^{*}$ \\
\hline Age (years; $n=64)$ & $39.57 \pm 10.13(21-60)^{*}$ & \\
\hline \multicolumn{3}{|l|}{ Gender } \\
\hline Male & $13(40.6)$ & $10(31.2)$ \\
\hline Female & $19(59.4)$ & $22(68.8)$ \\
\hline \multicolumn{3}{|l|}{ Marital status } \\
\hline Single & $7(21.9)$ & $3(9.4)$ \\
\hline Married & $20(62.5)$ & $22(68.7)$ \\
\hline Divorced/widowed & & $7(21.9)$ \\
\hline \multicolumn{3}{|l|}{ Educational level } \\
\hline None & $5(15.6)$ & $14(43.8)$ \\
\hline Primary & $8(25.0)$ & $6(18.7)$ \\
\hline$\geq$ Secondary & $19(59.4)$ & $12(37.5)$ \\
\hline \multicolumn{3}{|l|}{ Occupation } \\
\hline Employed & $26(81.2)$ & $22(68.7)$ \\
\hline Unemployed & $3(9.4)$ & $6(18.8)$ \\
\hline House wife & $3(9.4)$ & $4(12.5)$ \\
\hline
\end{tabular}

Table 2 Student t-test Analysis for Independent Samples on Quality of Life and CD4 cells of the Participants between Control and Experimental Groups at Baseline $(\mathrm{N}=82)$

\begin{tabular}{|c|c|c|c|c|}
\hline $\begin{array}{l}\text { Variables } \\
\text { Quality of life domains }\end{array}$ & Control group $n=4$ I & Exp. Group n=4 I & T-test value & P-value \\
\hline Physical functioning (\%) & $58.66 \pm 14.83$ & $58.29 \pm 16.68$ & 0.105 & 0.917 \\
\hline Role-Physical health (\%) & $40.98 \pm 33.56$ & $31.95 \pm 32.30$ & 1.24 & 0.114 \\
\hline Role-Emotional health (\%) & $47.15 \pm 35.74$ & $37.40 \pm 31.81$ & 1.305 & 0.096 \\
\hline Energy/fatigue & $59.76 \pm 10.93$ & $53.54 \pm|2.4|$ & 1.409 & 0.088 \\
\hline Emotional well being (\%) & $62.12 \pm|3.6|$ & $56.83 \pm 13.07$ & 1.796 & 0.076 \\
\hline Social functioning (\%) & $64.27 \pm 12.12$ & $65.37 \pm 12.86$ & 0.398 & 0.692 \\
\hline Bodily pain (\%) & $57.07 \pm 21.94$ & $55.37 \pm 23.78$ & 0.338 & 0.736 \\
\hline General health (\%) & $61.38 \pm 11.87$ & $51.14 \pm 12.09$ & I.87| & 0.063 \\
\hline CD4 counts (cells/mm 3 ) & $483.76 \pm 135.99$ & $493.07 \pm|44.5|$ & 0.301 & 0.764 \\
\hline
\end{tabular}

Table 3 Paired Student t-test Analysis on Quality of Life and CD4 Cell Counts of the Participants (Pre- and post-test) in the Experimental and control groups $(\mathrm{N}=64)$

\begin{tabular}{|c|c|c|c|c|}
\hline $\begin{array}{l}\text { Variables } \\
\text { Experimental Group }\end{array}$ & Pre-test $n=32$ & Post-test $n=32$ & t-test value & p-value \\
\hline Physical functioning (\%) & $60.73 \pm 15.62$ & $77.03 \pm 10.84$ & 7.385 & $0.000 *$ \\
\hline Role-Physical health (\%) & $48.54 \pm 30.82$ & $80.47 \pm 10.50$ & 8.434 & $0.000 *$ \\
\hline Role-Emotional health (\%) & $43.87 \pm 219.54$ & $85.43 \pm 16.78$ & 7.792 & $0.000 *$ \\
\hline Energy/fatigue (\%) & $56.19 \pm 13.22$ & $72.50 \pm 8.71$ & 8.841 & $0.000 *$ \\
\hline Emotional well being (\%) & $55.49 \pm|4.3|$ & $71.63 \pm 7.72$ & 8.263 & $0.000 *$ \\
\hline Social functioning $(\%)$ & $68.23 \pm 11.47$ & $77.19 \pm 9.33$ & 7.426 & $0.000 *$ \\
\hline Bodily pain (\%) & $54.61 \pm 22.92$ & $78.91 \pm 12.30$ & 8.715 & $0.000 *$ \\
\hline General health (\%) & $53.26 \pm 12.74$ & $72.92 \pm 7.90$ & 8.117 & $0.000 *$ \\
\hline $\begin{array}{l}\text { CD4 counts (cells/mm }{ }^{3} \text { ) } \\
\text { Control Group }\end{array}$ & $491.18 \pm 152.73$ & $672.25 \pm 227.59$ & 6.152 & $0.000 *$ \\
\hline Physical functioning (\%) & $57.81 \pm 17.11$ & $57.09 \pm 8.75$ & 0.648 & 0.745 \\
\hline Role-Physical health (\%) & $41.03 \pm 31.42$ & $41.38 \pm 24.35$ & 0.422 & 0.831 \\
\hline Role-Emotional health (\%) & $46.77 \pm 34.68$ & $41.66 \pm 23.96$ & $\mathrm{I} .497$ & 0.46 \\
\hline Energy/fatigue (\%) & $59.09 \pm 12.82$ & $55.32 \pm 9.62$ & 3.151 & $0.036 *$ \\
\hline Emotional well being (\%) & $61.44 \pm 15.32$ & $55.19 \pm 8.86$ & 4.138 & $0.009 *$ \\
\hline Social functioning (\%) & $63.71 \pm 13.27$ & $56.09 \pm 7.48$ & 4.839 & $0.004 *$ \\
\hline Bodily pain (\%) & $52.38 \pm 20.44$ & $50.31 \pm 14.48$ & 1.462 & 0.596 \\
\hline General health (\%) & $60.89 \pm 14.25$ & 55.75..99.09 & 3.402 & $0.026 *$ \\
\hline CD4 counts (cells $/ \mathrm{mm}^{3}$ ) & $489.52 \pm 108.78$ & $522.66 \pm 123.06$ & 3.837 & $0.001 *$ \\
\hline
\end{tabular}

*Significant at $\mathrm{p}<0.05$. 
Table 4 Student t-test Analysis for Independent Samples (between the Control and Experimental Groups at the end of the Study, i.e. Post-test) on Quality of Life and CD4 Cell Counts of the Participants $(\mathrm{N}=64)$

\begin{tabular}{lllll}
\hline Variables & Control Group n=32 & Exp. Group n=32 & t-test value & p-value \\
\hline Physical functioning (\%) & $57.09 \pm 8.75$ & $77.03 \pm 10.84$ & 8.099 & $0.000 *$ \\
Role-Physical health (\%) & $41.38 \pm 24.35$ & $80.47 \pm 10.50$ & 8.355 & $0.000 *$ \\
Role-Emotional health (\%) & $41.66 \pm 23.96$ & $85.43 \pm 16.78$ & 8.463 & $0.000 *$ \\
Energy/fatigue & $55.32 \pm 9.62$ & $72.50 \pm 8.71$ & 7.492 & $0.000 *$ \\
Emotional well being (\%) & $55.19 \pm 8.86$ & $71.63 \pm 7.72$ & 7.911 & $0.000 *$ \\
Social functioning (\%) & $56.09 \pm 7.48$ & $77.19 \pm 9.33$ & 9.978 & $0.000 *$ \\
Bodily pain (\%) & $50.31 \pm 14.48$ & $78.91 \pm 12.30$ & 8.516 & $0.000 *$ \\
General health (\%) & $55.75 . \pm 9.09$ & $72.92 \pm 7.90$ & 8.062 & $0.000 *$ \\
CD4 counts (cell//mm & $522.66 \pm 123.06$ & $672.25 \pm 227.59$ & 3.271 & $0.002 *$ \\
\hline
\end{tabular}

*Significant at $\mathrm{p}<0.05$.

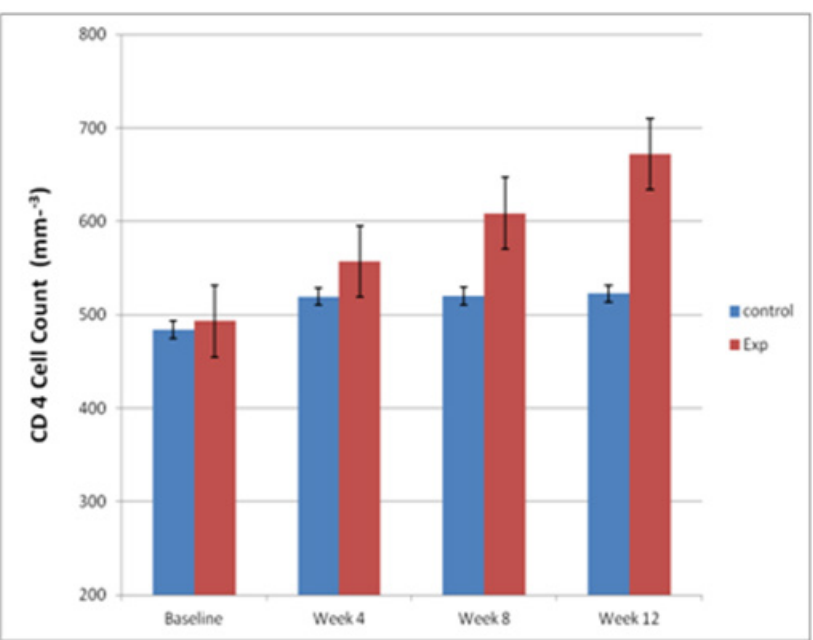

Figure I CD4 cell counts of the participants from the baseline to the I2th week of the study.

\section{Discussion}

This study examined effect of moderate intensity treadmill aerobic exercise on quality of life and CD4 cell counts of HIV infected persons in Nigeria. To our knowledge based on literature search, this seems to be the first study from this part of the world to showcase the beneficial impact of aerobic exercise on quality of life and CD4 cell counts of HIV seropositives on HAART.Quality of life was evaluated under eight domains using RAND 36-item short form (SF-36) Health Survey Questionnaire.The baseline values of the domains of quality of life and CD4 cells in the experimental and control groups showed no significant difference ( $p>0.05$ ), inferring homogeneity of these groups at the inception of the aerobic exercise training program. The result indicated significant increase $(\mathrm{p}<0.05)$ in the scores of the eight domains between pre - and post - tests in the experimental group. In the control group, there was no significant change ( $p>0.05)$ in the scores of four out of the eight domains (physical functioning, role limitation due to physical health, role limitation due to emotional health and bodily pain) between pre-and post-tests. In the remaining four domains (energy/fatigue, emotional well being, social functioning and general health), significant reduction $(\mathrm{p}<0.05)$ in the scores was observed at the end of the study. Comparing the scores of the experimental group with those of the control group at the end of the study showed significant difference $(p<0.05)$ in favor of the former in all the eight domains of quality of life. The finding of this study also revealed significant increase $(p<0.05)$ in the scores of both physical (physical functioning, role limitation due to physical health, bodily pain and general health) and mental (limitation due to emotional health, energy/fatigue, emotional well being and social functioning) components of SF-36 quality of life questionnaire among the participants in the experimental group at the end of the study. The scores on these components increased by $+42.5 \%$ and $+37.1 \%$ respectively from the baseline, while the scores of the participants in the control group decreased by $-3.6 \%$ and $-10.0 \%$ respectively at the end of the study. The finding also indicated that the participants in the experimental group exhibited positive trends in the scores of the eight domains of the SF-36 quality of life scales, while the control group demonstrated negative trends in four out of the eight domains.

This finding corroborates that of Tiozzo et al. ${ }^{19}$ which demonstrated significant improvements in both physical and mental components of SF-36 quality of life scales (although to a lesser degree) in the values of 16 and $9 \%$ respectively after 12 weeks of combined aerobic and resistance exercise program among experimental participants. The authors reported lower scores on the same scales (-18 and $-12 \%$ respectively) among the control participants. Tiozzo et al. ${ }^{19}$ also reported positive trends in seven of the eight scales among the participants in the exercise training intervention group, while the control participants exhibited negative trends in the same SF-36 scales. These discrepancies in scores and trends observed between the finding of this study and that of Tiozzo et al..$^{19}$ could be attributed to geographical and racial differences. Tiozzo et al. ${ }^{19}$ conducted their study in Miami, United State of America among African Americans, Hispanics and non Hispanics, this study was conducted in Maiduguri, Borno State, Nigeria among Nigerians at an epoch of insurgency in that part of the country. It could be that the exercise intervention impacted greatly and positively among the participants by reducing the tension and stress generated by the insurgency. Our finding also supports that of Stringer et al. ${ }^{24}$ which reported that 6 weeks of aerobic training significantly increased quality of life scores of HIV infected persons. It is also in agreement with that of LaPerriere et al. ${ }^{22}$ which found that aerobically trained HIV infected men were protected from stress-related impairments in immune status compared to sedentary controls. This assertion holds true in that the experimental group in this present study exhibited higher CD4cell counts at the end of the study compared to the control group that did not participate in the exercise training program. Orlando et al. ${ }^{9}$ established that HIV infected persons have higher episodes of psychological disorders which negatively alters their quality of life continuum compared to non infected individuals. Conversely, the finding of this study is at variance with that of Birk et al., ${ }^{25}$ which recorded no significant improvement in quality of life of the participants following 12 weeks of combined massage and exercise treatment. This variation may be attributed to the differences in the modes/types of intervention used in both studies. The present study employed moderate intensity treadmill aerobic exercise without massage, while the type of exercise treatment utilized by Birk et al. ${ }^{25}$ was not specified. 
As stated at the beginning of this discussion, the CD4 cell counts of the control and intervention groups were similar at baseline $(\mathrm{p}>0.05)$, that is before the commencement of the 12 weeks aerobic exercise training program. At the end of the study, significant improvement $(p<0.05)$ in CD4 cell counts was observed between pre-and posttests in the experimental group. Significant difference $(p<0.05)$ in this variable was also recorded between the experimental and control groups at the same period. In the control group, the CD4cell counts showed significant increase $(p<0.05)$ from pre - test to post - test, although not as significant as that observed in the experimental group. The finding of this study on CD4cell counts corroborates those of some past studies which reported significant improvement of this variable post exercise intervention, and partially supports others which found stability or insignificant improvements after exercise therapy. Several studies ${ }^{35,16,36,37}$ reported increases in CD4cell counts in HIV seropositives, while others ${ }^{38,39,40}$ found stability in CD4cell counts after exercise training program. Terry et al. ${ }^{39}$ documented non statistical significant increase in CD4cell counts of HIV infected persons after 12 weeks moderate intensity aerobic exercise. A study by Tiozzo et al. ${ }^{19}$ revealed an insignificant reduction $(-3 \%$ from baseline) of CD4 cell counts in the experimental group participants, while the control group experienced a significant reduction of $-16 \%$ after 12 weeks of combined aerobic and resistance exercise. In other words, the CD4cells of the experimental group were more or less stable compared to those observed in the control group. A significant increase of $27.18 \%$ in CD4 cell counts was reported by Ezema et al. ${ }^{37}$ among exercise group participants, while the non exercise group recorded an insignificant increase of $3.60 \%$ in CD4 cell counts. The finding of this present study indicated $36.9 \%$ significant increase in CD4 cell counts in the experimental group, while in the control group, the CD4 cell counts improved by $6.8 \%$, corroborating that of Ezema et al. ${ }^{37}$ Mustafa et al. ${ }^{41}$ observed that HIV seropositives' self-reporting exercise participation had $107.5 \%$ higher CD4 cell counts compared to non exercise participants. The exercise group also presented with slower disease progression to AIDS, less symptoms and decreased rates of mortality compared to non exercisers. Refusal to participate in prescribed exercise training programs had been linked to lower CD4 cell count levels among HIV population. ${ }^{41}$

According to LaPerreire et al. ${ }^{16}$ stress reduces immunological variables, especially CD4 cell counts. In addition, anxiety and depression are the most common symptoms experienced by HIV infected population and indicators of low quality of life with concomitant stress. ${ }^{12,10}$ Thus, the considerableimprovement in CD4 cell counts observed in the experimental group in this study could be ascribed somewhat to the significant increase in the scores of the domains of quality of life of this group at the end of the study compared to the baseline scores. In essence, there seems to be a direct relationship between CD4 cell counts and state of mental wellbeing which is a continuum of quality of life. It might also be that the significant increase in this particular variable in the experimental group was due to the ability of aerobic exercise training to stimulate the formation of certain antibodies that prevent glycoprotein (gp) 120 (potent HIV protein molecules) from attaching to receptor sites of CD4 cells. ${ }^{28}$ By this process, the damaged immune system is reconstituted and HIV disease progression to AIDS is slowed down. ${ }^{29}$ This is quite similar to the role HAART plays in HIV infection. The CD4 cell counts also significantly improved in the control group who did not participate in the 12 weeks aerobic exercise training program. It could be recalled that the authors organized lectures for this group once a week on issues pertaining to nutrition, meaning of HIV and AIDS, relationship and differences between HIV and AIDS, mode of HIV transmission and its prevention, and adherence to ARV therapy. The authors deduced that the participants in this group might have applied what they learnt from the lectures to practice, especially on nutrition and adherence to therapy. Good nutrition improves immune health, and Penzak et al. ${ }^{13}$ established an association between depression and altered course of infection due to impaired immune function and non adherence to therapy. The participants in the control group also selfreported/admitted that the lectures they received made them to feel and sleep better. Having enough sleep improves and boosts immune functions. In the study of Tiozzo et al. ${ }^{19}$ the control participants did not receive any lecture, but rather, they were telephoned every four weeks in order to maintain contact and promote their interest in the study. This might have attributed to the significant reduction (-16\%) in CD4 cell counts recorded in that group of participants.

\section{Limitations}

Our participants on HAART were enrolled from an ARV clinic located at a tertiary health facility which is a teaching hospital in the Northeastern Nigeria, this sample do not essentially signify the general Nigerian HIV seropositive population on HAART. The nutritional intake of the participants was beyond our control, so we did not assess this. We cannot therefore really establish to what extent the diet of these participants affected the variables (especially the CD4 cells) investigated in this study. However, the homogeneous values of these variables at baseline might have somehow assuaged this particular limitation.

\section{Conclusion}

The finding of this study has showcased that exercise is beneficial to the general health and wellbeing of HIV population, and therefore corroborates the findings already reported by other studies mostly conducted in the developed world. It also upholds the fact that no study has so far reported deterioration in the health status of HIV seropositives on HAART as a consequence of moderate intensity exercise. The lectures giving to the control group participants seemed to have acted as psychological boost. This group self-reported that they felt good and slept better as a result of the lectures.Contacting the non exercise group on phone may not be enough, scholars should be encouraged in addition, to organize lectures on relevant issues pertaining to HIV and AIDS for this group of participants.

\section{Acknowledgments}

We sincerely thank the participants who out of their tight schedules took part in this study. We also appreciate the efforts of the staff of the antiretroviral clinic where the participants enrolled for the study. Finally, we thank Medical Education Partnership Initiatives in Nigeria (MEPIN) for supporting and sponsoring this study. In essence, this is a part of a project supported by Medical Education Partnership Initiatives in Nigeria (MEPIN) project funded by Fogarty International Center, the Office of AIDS Research, and the National Human Genome Research Institute of the National Institute of Health, the Health Resources and Services Administration (HRSA) and the Office of the U.S. Global AIDS Coordinator under the Award Number R24TW008878. The content is solely the responsibility of the authors and does not necessarily represent the official views of the funding body.

\section{Conflicts of interest}

None.

\section{References}

1. Nasidi A, Harry TO, Ajose-Coker OO. Evidence of LAV/HTLVII infection and AIDS related complex in Lagos. Nigeria. II international Conference on AIDS, Paris France 23-25. 1986. 
2. Mafeni JO and Fajemisin OA. HIV/AIDS in Nigeria: situation, response and Prospects, Key Issues . Policy Project, Nigeria, 2003

3. Orubuloye IO, Oguntimehin F. Death is pre-ordained, it will come when it is due: attitude of men to death in the presence of AIDS in Nigeria. Resistances to Behavioural change to Reduce HIV/AIDS infection. 1999;101-111.

4. Caldwell JC, Orubuloye IO, Caldwell P. Under reaction to AIDS in SubSaharan Africa. Social Science Medicine. 1992;34(11):1169-1182.

5. The World Bank. World DataBank, World Development Indicators. 2011.

6. Federal Ministry of Health (FMOH). Technical report on National HIV Sero-prevalence sentinel Survey among Pregnant Women Attending Antenatal Clinics in Nigeria. Abuja. 2010.

7. Nattrass N. The moral economy of AIDS in South Africa. Cambridge University Press. 2004;54:166-167.

8. Phaladze NA, Humans S, Dlamini SB, et al. Quality of life and the concept of "living well" with HIV/AIDS in sub Saharan Africa. Journal of Nursing Scholarship. 2005;32(2):120-126.

9. Orlando M, Burnam MA, Beckman R, et al. Re-Estimating the Prevalence of Mental Health Conditions in a National Sample of Persons Under Care for HIV: Results from the HIV Cost and Services Utilization Study. International Journal of Methods in Psychiatric Research. 2002;11(2):75-82.

10. Rivero-Mendez M, Portillo C, Solis-Baez SS, et al. Symptoms and quality of life for people living with HIV infection in Puerto Rico. $P R$ Health Sci J. 2009;28(1):54-59.

11. Cabaj RP. Management of anxiety and depression in HIV-infected patients. J Int Assoc Physicians in AIDS Care. 1996;2(6):11-16.

12. Heckman TG, Heckman BD, Kochman A, et al. Psychological symptoms among persons 50 years of age and older living with HIV disease. Aging Ment Health. 2002;6(2):121-128.

13. Penzak SR, Reddy YS, Grimsley SR. Depression in patients with HIV infection. Am J Health Syst Pharm. 2000;57(4):376-386.

14. World Health Organization. HIV/AIDS: Global Health Observatory. 2011.

15. Jaggers JR. Clinical exercise, stress, and HIV infection: identifying the associations and testing the effects. Dissertation submitted in partial fulfillment of the requirements for the degree of doctor of philosophy in exercise science, Arnold School of Public, Health University of South Carolina. 2010.

16. LaPerriere A, Antoni MH, Ironson G, et al. Effects of aerobic exercise training on lymphocyte subpopulations. Int J Sports Med. 1994;15(3):127-130.

17. Nokes KM, Kendrew J. Correlates of sleep quality in persons with HIV disease. J Assoc Nurses AIDS Care. 2001;12(1):17-22.

18. Darko DF, Mitler MM, White JL. Sleep disturbance in early HIV infection. Focus. 1995;10(11):5-6.

19. Tiozzo E, Jayaweera D, Rodriguez A, et al. Short-term combined exercise training improves the health of HIV-infected patients. Journal of AIDS and HIV Research. 2013;5(3):80-89.

20. Boufassa F, Dulioust A, Lascaux AS, et al. Lipodystrophy in 685 HIV-1 treated patients: influence of antiretroviral treatment and immunovirological response. HIV Clin Trials. 2001;2(4):339-345.

21. O’Brien K, Nixon S, Tynan AM, et al. Aerobic Exercise Interventions for People Living with HIV/AIDS: Implications for Practice, Education, and Research. Physiotherapy Canada. 2006;58:114-129.

22. LaPerriere AR, Antoni MH, Schneiderman N, et al. Exercise intervention attenuates emotional distress and natural killer cell decrements following notification of positive serologic status for HIV-1. Biofeedback and Self-regulation. 1990;15(3):229-242.
23. Mutimura E, Stewart A, Crowther NJ, et al. The effects of exercise training on quality of life in HAART treated HIV-positive Rwandan subjects with body fat. Qualil Life Res. 2008;17(3):377-385.

24. Stringer WW, Berezovskaya M, O’Brien WA, et al. The effect of exercise training on aerobic fitness, immune indices, and quality of life in HIV+ patients. Med Sci Sports Exerc. 1998;30(1):11-16.

25. Birk TJ, McGrady A, MacArthur RD, et al. The effects of massage therapy alone and in combination with other complementary therapies on immune system measure and quality of life in human immunodeficiency virus. J Altern Complement Med. 2000;6(5):405-414.

26. Ciccolo JT, Jowers EM, Bartholomew JB. The Benefits of Exercise Training for Quality of Life in HIV/AIDS in the Post-HAART Era. Sports Med. 2004;34(8):487-499.

27. Ramirez-Marrero FA, Smith BA, Melendes-Brau N, et al. Physical and leisure activity, body composition and lifestyle in HIV-positive Hispanics in Puerto Rico. J Nurses AIDS Care. 2004;15(4):68-77.

28. Veljkovic V. Can exercise help prevent and treat AIDS? The Institute of Science in Society (ISIS) report .2005;pm;13/4/12.

29. Djordjevic A, Veljkovic M, Antoni S, et al. The presence of antibodies recognizing a peptide derived from the second conserved region of HIV-1 gp120 correlates with non-progressive HIV infection. Curr HIV Res. 2007;5(5):443-448.

30. Calitz M. The effect of an aerobic exercise program on the health-related quality of life of HIV positive employees. A dissertation submitted in fulfillment of the requirements for the degree, Magister Artium (Human Movement Sciences) in the Department of Biokinetics, Sport and Leisure Sciences at the University of Pretoria, Faculty of Human Sciences. 2008

31. Tsai C, Bayliss MS, Ware JE. SF-36® Health Survey Annotated Bibliography: Second Edition (1988-1996). Boston, MA: Health Assessment Lab, New England Medical Center. 1997.

32. Ware JE, Snow KK, Kosinski M, et al. SF-36® Health Survey Manual and Interpretation Guide. Boston, MA: New England Medical Center, the Health Institute. 1993.

33. Mc Horney CA, Ware JE, Raczek AE. The MOS 36-Item ShortForm Health Survey (SF-36®) II Psychometric and clinical tests of validity in measuring physical and mental health constructs. Med Care. 1993;31(3):247-263.

34. Burgess A, Dayer M, Catalan J, et al. The reliability and validity of two HIV-specific health related Quality of Life measures: A preliminary analysis. AIDS. 1993; 7:1001-1008.

35. LaPerriere AR, Fletcher MA, Antoni MH, et al. Aerobic exercise training in an AIDS risk group. Int J Sports Med. 1991;12(1):S53-S57.

36. Perna FM, LaPerriere A, Klimas N, et al. Cardiopulmonary and CD4 cell changes in response to exercise training in early symptomatic HIV infection. Med Sci Sports Exerc. 1996;31(7):973-979.

37. Ezema CI, Onwunali AA, Lamina S, et al. Effect of aerobic exercise training on cardiovascular parameters and CD4 cell count of people living with human immunodeficiency virus/acquired immune deficiency syndrome: A randomized controlled trial. Nigerian Journal of Clinical Practice. 2014;17(5):543-548

38. Schneiderman N, Fletcher M, Meijer OG, et al. Cardiopulmonary and CD4 cell changes in response to exercise training in early symptomatic HIV infection. Med Sci Sports Exerc. 1999;31(7):973-979.

39. Terry L, Sprinz E, Ribeiro JP. Moderate and high intensity exercise training in HIV-1 seropositive individuals: A randomized trial. Int JSports Med. 1999;20:142-146.

40. Mustafa T, Sy FS, Macera CA, et al. Association between exercise and HIV disease progression in a cohort of homosexual men. Annals of Epidemiology. 1999;9(2):127-131

41. MacArthur RD, Levine SD, Birk TJ. Supervised exercise training improves cardiopulmonary fitness in HIV infected persons. Med Sci Sports Exerc. 1993;25(6):684-688. 